\title{
High Power, Low Threshold, Raman DFB Fibre Lasers
}

\author{
Jindan Shi, Shaif-ul Alam, Morten Ibsen \\ Optoelectronics Research Centre, University of Southampton, Highfield, Southampton, SO17 1BJ, UK \\ jxs@orc.soton.ac.uk
}

\begin{abstract}
We demonstrate highly efficient Raman gain based distributed-feedback fibrelasers at $\sim 1.11 \mu \mathrm{m}$ with up to $2 \mathrm{~W} \mathrm{CW}$ output-power with $<0.01 \mathrm{~nm}$ linewidth. The lasers are $30 \mathrm{~cm}$ long and UV-written directly into two types of passive germanosilicate fibres.
\end{abstract}

\section{Introduction}

All-fibre rare-earth doped distributed feedback (DFB) lasers have attracted a lot of interest in recent years [1]. In addition to providing inherent fibre compatibility thereby ensuring low insertion loss, they exhibit numerous excellent optical properties. These include, outstanding beam quality, very low noise characteristics and extremely narrow linewidth, making them very useful sources in a number of applications in telecommunications, range-finding and LIDAR, and sensing [2-4]. It is a prerequisite of these sources to have high-concentration of rare-earth ions doping in the core of the fibre to facilitate high efficiency. High concentration of rare-earth ions can lead to problems with output power stability due to pair-ion quenching leading to inefficient pump energy transfer and excessive thermal loading. The operating wavelengths are also limited to regions characteristic to the specific rare-earth material used. A Raman gain based DFB fibre laser could overcome these limitations whilst maintaining all the attractive optical properties. This would therefore open up the prospect of generating high-power narrow linewidth low-noise oscillation at any desired wavelength ranging from the visible to the infrared region, pending only the availability of a suitable pump source [5-6]. Theoretical studies suggest that Raman DFB fibre lasers could be made comparatively efficient by employing $\pi$ phase-shifted fibre Bragg gratings of only a few tens of centimetres of passive germanium doped silica fibres, and also that these should exhibit only watt-level threshold [7-8]. Only recently an experimental demonstration of Raman DFB fibre laser operating at $1.58 \mu \mathrm{m}$ was reported [9]. The threshold power required for laser oscillation in that case was extremely high at $40 \mathrm{~W} @ 1480 \mathrm{~nm}$, and only $65 \mathrm{~mW}$ of output power was obtained at a pump power of $80 \mathrm{~W}$, corresponding to a conversion efficiency of only $0.08 \%$.

In this work, we experimentally demonstrate $30 \mathrm{~cm}$ long Raman DFB (R-DFB) lasers in high-NA (0.35) and standard-NA (0.12) passive germanosilica $(\mathrm{Ge} / \mathrm{Si})$ fibres with threshold powers in the low watt regime. Up to $2 \mathrm{~W}$ of $\mathrm{CW}$ output power is obtained for a pump power of only $13.7 \mathrm{~W}$ with a threshold power of only $\sim 1 \mathrm{~W}$. In addition to being the highest efficiency R-DFB laser demonstrated to date, it is also the highest ever reported output power from any DFB fibre laser, incl. rare-earth doped DFB fibre lasers, to the best of our knowledge. The highest achieved output power corresponds to a conversion efficiency of $14.6 \%$ which is $\sim 182$ times higher than the R-DFB reported in [9] The slope efficiencies with respect to the absorbed pump power are $\sim 92 \%$ for the high-NA fibre, and $\sim 74 \%$ for the low-NA fibre, respectively. The R-DFB fibre lasers oscillate with a linewidth of $<0.01 \mathrm{~nm}$ at $\sim 1.11 \mu \mathrm{m}$ and the signal-to-noise ratio (SNR) is in excess of $55 \mathrm{~dB}$ for all output power levels.
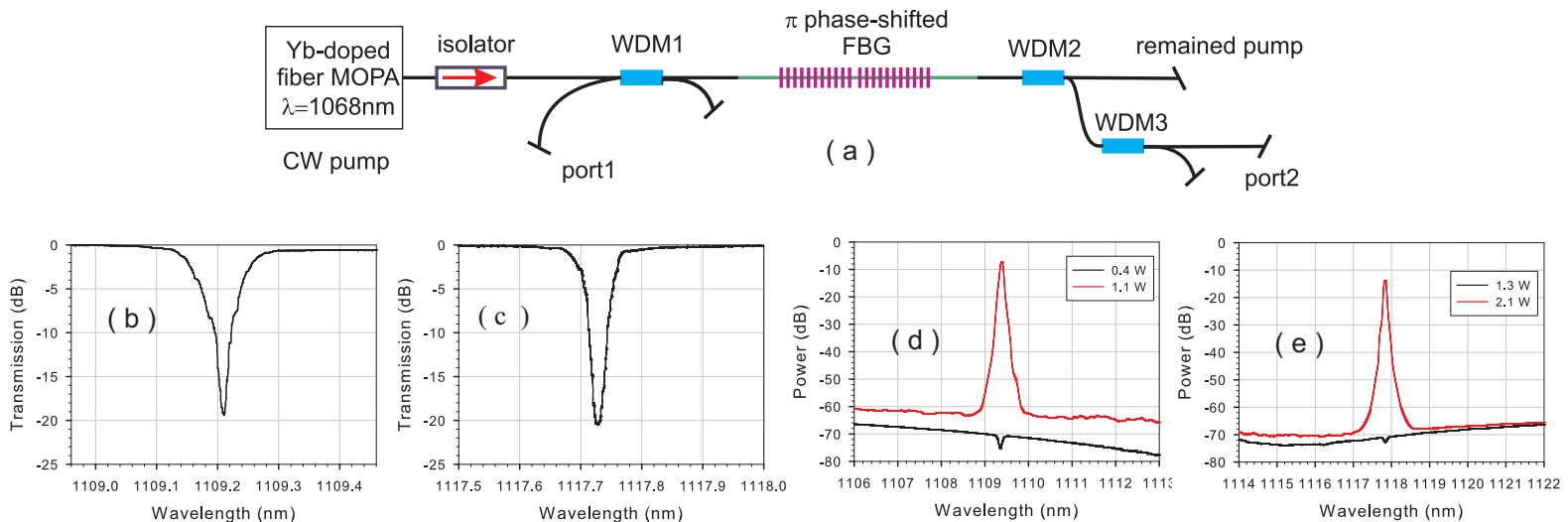

Fig. 1 (a) Schematic diagram of the experimental setup; Passive cold cavity transmission spectra of Bragg gratings in (b) R-DFB1, and (c) R-DFB2, measured with a resolution bandwidth of $0.01 \mathrm{~nm}$. Forward DFB output spectra just below and just above threshold for (d) R-DFB1, and (e) R-DFB2, measured with a resolution bandwidth of $0.1 \mathrm{~nm}$. 


\section{Experimental setup and results}

The schematic diagram of the experimental setup used to generate and analyse the R-DFB fibre laser oscillation is shown in Fig. 1(a). A CW, 1068nm, Yb-doped all-fibre MOPA with a maximum output power of 15W is used as the Raman pump source. The output of the source is spliced to a $1064 \mathrm{~nm} / 1117 \mathrm{~nm}$ wavelength division multiplexer (WDM1) which in turn is spliced to the $\pi$ phase-shifted DFB grating. The forward output of the DFB grating is spliced to two cascaded $1064 \mathrm{~nm} / 1117 \mathrm{~nm}$ WDMs (WDM2 and WDM3) to better isolate the forward DFB fibre signal from any residual pump power. The total output power and the spectra of the R-DFB fibre lasers are measured from port1 (for backward operation) and port2 (for forward operation). All fibre ends are angle-cleaved to prevent end-feedback, and all components (WDMs and DFB gratings) are mounted on heat sinks to help control the temperature and better remove any generated heat.

Two types of commercially available Ge/Si fibres, being the PS980 fibre from Fibercore Ltd. and the UHNA4 fibre from Nufern, are selected because of their single transverse-mode properties in the region of the projected Raman oscillation with the $1068 \mathrm{~nm}$ pump source. Table 1 lists the key parameters of the fibres including the NA, the mode field diameter (MFD) at $1100 \mathrm{~nm}$ and the unpolarised peak Raman gain coefficients which are estimated by considering the concentration of Germanium doping only [10]. It is worth noting that the PS980 fibre in fact is a Boron co-doped Ge/Si fibre and that the Raman gain coefficient therefore likely is slightly higher than that of a Germanium-only doped fibre of similar NA.

The DFB gratings are $30 \mathrm{~cm}$ long with centre $\pi$ phase-shifts to help facilitate the lowest possible lasing threshold. They are written directly into the fibres without the aid of hydrogen loading with 244nm CW UVlight using the continuous grating writing technique [11]. The passive cold cavity phase-shifted grating transmission spectra in the two fibres are shown in Fig. 1(b) and Fig. 1(c). From uniform test-gratings written prior to the phase-shifted DFB gratings, the coupling coefficients, $\kappa$, of the gratings are nearly identical with a value of $37 \mathrm{~m}^{-1}$. Due to the low refractive index modulation in the gratings $\left(\sim 10^{-5}\right)$ and the absence of photosensitising loading gas, the grating inscription-induced loss in the two fibres is minimal. The total propagation loss is therefore not much higher than the linear loss of the pristine fibres, which is $\sim 20 \mathrm{~dB} / \mathrm{km}$ for PS 980 and $\sim 5 \mathrm{~dB} / \mathrm{km}$ for UHNA4 at $\sim 1 \mu \mathrm{m}$. Table 1 also summarizes the key Bragg grating parameters used for the R-DFB lasers.

Table1: Key parameters of the fibres and Bragg gratings used for the Raman DFB fibre lasers.

\begin{tabular}{|c|c|c|c|c|c|c|c|c|}
\hline R-DFB & Fibre & NA & $\begin{array}{c}\text { MFD } \\
\text { @1100nm } \\
(\mu \mathrm{m})\end{array}$ & $\begin{array}{c}g_{r} \\
@ 1.06 \mu \mathrm{m} \\
\left(\mathbf{1} 10^{-13} \mathrm{~m} / \mathrm{W}\right)\end{array}$ & $\begin{array}{c}\lambda_{B} \\
(\mathbf{n m})\end{array}$ & $\begin{array}{c}\boldsymbol{k} \\
\left(\mathbf{m}^{-1}\right)\end{array}$ & $\begin{array}{l}L_{\mathrm{DFB}} \text { gratings } \\
\quad(\mathrm{cm})\end{array}$ & $\begin{array}{c}L_{\text {total }} \\
\text { (incl. } \\
\text { pigtails) } \\
\text { (cm) }\end{array}$ \\
\hline 1 & UHNA4 & 0.35 & $2.6 \pm 0.3$ & 1.55 & 1109.21 & 37 & 30 & 61 \\
\hline 2 & PS980 & 0.12 & $6.2 \pm 0.3$ & 0.86 & 1117.73 & 37 & 30 & 38 \\
\hline
\end{tabular}

\section{Results and Discussions}

Fig. 1(d) and Fig. 1(e) shows the forward DFB output spectra just below, and just above, the lasing threshold for the two fibres. As is clearly evident the lasing occurs exactly at the Bragg wavelength of the phase shifted grating as expected from a $\pi$ phase-shifted grating structure. The threshold power for lasing is $\sim 1 \mathrm{~W}$ and $\sim 2 \mathrm{~W}$ for the high-NA (Fig. 1(d)) and standard-NA (Fig. 1(e)) fibres, respectively. Evidently, the lower lasing threshold is observed in R-DFB1 because of the higher Raman gain coefficient of the high-NA fibre. This indicates that even lower lasing threshold power should be possible by employing higher Raman gain coefficient nonlinear fibres such as for example tellurite glass based non-silica fibre [12]. Both lasers exhibit more than $55 \mathrm{~dB}$ SNR soon after the lasing action takes place, and their $3 \mathrm{~dB}$ linewidths are measured to be less than $0.01 \mathrm{~nm}$ with a high resolution OSA (Advantest Q8384). More accurate linewidth measurements can be carried out by using for example delayed self-heterodyne or self-homodyne methods as also demonstrated in [9].

The evolution of the total R-DFB output powers with respect to the incident (launched) pump powers and converted pump powers is shown in Fig. 2(a) and Fig. 2(b), respectively. The maximum output power obtained is $\sim 2 \mathrm{~W}$ for an incident pump power of $13.7 \mathrm{~W}$ for R-DFB1, and $\sim 1.5 \mathrm{~W}$ for an incident pump power of $15 \mathrm{~W}$ for R-DFB2. The uncertainty in the measured output power is estimated to be $\pm 5 \%$, due to the use of a thermal power metre. For both R-DFB lasers, the output power grows slowly for low incident pump powers, but gradually increases as the pump power is increased (as seen in Fig. 2(a)). Although not a particularly good measure of the efficiency due to the nonlinear nature of the output power-growth with pump power, the output powers are seen to increase with slope efficiencies above $13 \%$ in both lasers for pump powers of $\sim 3 \mathrm{~W}$ above threshold. This trend is slightly lower than predicted by simulations but could in part be explained by the presence of minor phase and amplitude errors in the gratings [8]. A better picture of the efficiencies is achieved 
by viewing the slope efficiency of the output power against converted pump power. The converted pump-power is measured by comparing the incident pump power with the remained pump power at the output of the DFB gratings as shown in Fig. 1(a). As seen from Fig. 2(b), the slope efficiencies are found to be $\sim 92 \%$ for R-DFB1 and $\sim 74 \%$ for R-DFB2. The reason for the lower slope-efficiency from R-DFB2 might be due to the slightly higher propagation loss in this fibre. However, the $92 \%$ in R-DFB1 is close to the theoretical maximum quantum conversion efficiency, which is in good agreement with numerical simulations. Fig. 2(c) and Fig. 2(d) illustrate the forward and backward output spectra at the maximum output power for R-DFB1 and R-DFB2, respectively. The spectra demonstrate that the high SNR and narrow-bandwidth properties are maintained even at the maximum output power level. A slight bandwidth broadening is observed when comparing the forward output spectra with those of the backward spectra, and in the case of the high-NA fibre the SNR is also observed to be slightly reduced although remains above $55 \mathrm{~dB}$. The reason for the slight broadening and reduction of SNR is believed to be due to co-propagating non-linear interplay between the forward signal and the residual pump power [13]. We hope to report further and give more detailed analysis on these observations in future publications.
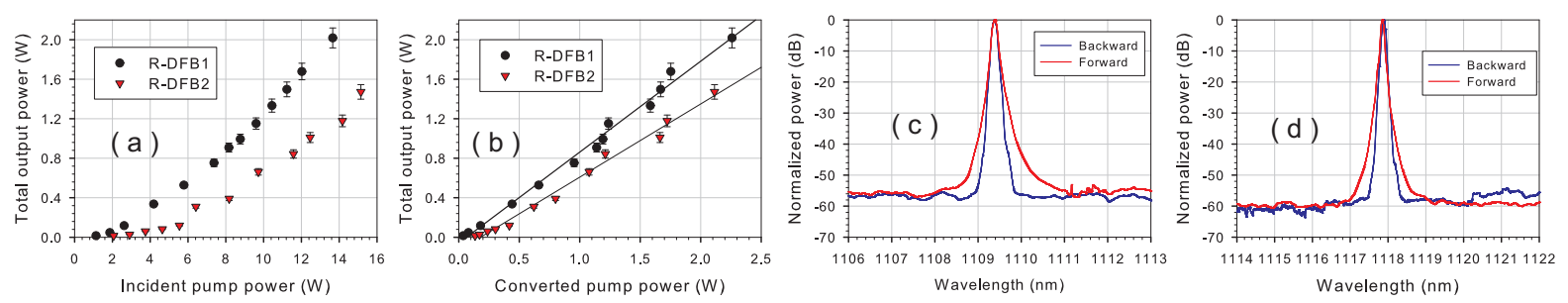

Fig. 2 Total R-DFB output power, (a) against incident pump power, and (b) against converted pump power. Normalised forward and backward DFB spectra at maximum output power of (c) R-DFB1, and (d) R-DFB2, measured with a resolution bandwidth of $0.1 \mathrm{~nm}$.

\section{Conclusion and Future work}

To conclude, we have experimentally demonstrated highly efficient Raman DFB fibre laser oscillation at $\sim 1.11 \mu \mathrm{m}$ from $30 \mathrm{~cm}$ centre phase-shifted Bragg gratings written in two types of passive Ge/Si fibres. The lasers are pumped with up to $15 \mathrm{~W}$ of $\mathrm{CW}$ power at $\sim 1.07 \mu \mathrm{m}$ to give narrow linewidth $\mathrm{CW}$ signal output powers in excess of $1.5 \mathrm{~W}$ in each case. The incident threshold pump power is as low as $\sim 1 \mathrm{~W}$ in a fibre with an NA of 0.35 , and only $\sim 2 \mathrm{~W}$ for a fibre with a standard NA of $\sim 0.12$. Our simulations indicate that even shorter cavity Raman DFB fibre lasers with sub-watt lasing threshold should be possible by employing high-index nonlinear fibres such as for example tellurite or chalcogenide glass based non-silica fibres as the host for the DFB gratings. We hope to report on the results of Raman DFB lasers in these types of fibres in future publications.

\section{References}

[1] J. T. Kringlebotn, J.-L. Archambault, L. Reekie, and D. N. Payne, "Er ${ }^{3+}: \mathrm{Yb}^{3+}$-codoped fiber distributed-feedback laser," Opt. Lett., 19, 2101-2103 (1994).

[2] M. Ibsen, E. Rønnekleiv, G. J. Cowle, M. O. Berendt, O. Hadeler, M. N. Zervas, and R. I. Laming, "Robust high power (>20 mW) allfibre DFB lasers with unidirectional and truly single polarisation outputs," in proceedings of CLEO '99, paper CWE4 (1999).

[3] M. Ibsen, A. Shaif-ul, M. N. Zervas, A. B. Grudinin, and D. N. Payne, "8- and 16-channel all-fiber DFB laser WDM transmitters with integrated pump redundancy," Photon. Technol. Lett., 11, 1114-1116 (1999).

[4] O. Hadeler, M. Ibsen, and M. N. Zervas, "Distributed-feedback fiber laser sensor for simultaneous strain and temperature measurements operating in the radio-frequency domain," Appl. Opt. 40, 3169-3175 (2001).

[5] K. O. Hill, B. S. Kawasaki, and D. C. Johnson, "Low-threshold cw Raman Laser," Appl. Phys. Lett., 29, 181-183 (1976).

[6] V. E. Perlin and H. G. Winful, "Distributed feedback fiber Raman laser," J. Quantum. Elec., 37, 38-47 (2001).

[7] Y. Hu and N. G. R. Broderick, "Improved design of a DFB Raman fibre laser," Optics Communications, 282, 3356-3359 (2009).

[8] J. Shi and M. Ibsen, "Effects of Phase and Amplitude Noise on $\pi$ Phase-Shifted DFB Raman Fibre Lasers," in proceedings of BGPP'10, paper JThA30 (2010).

[9] P. S. Westbrook, K. S. Abedin, J. W. Nicholson, T. Kremp, and J. Porque, "Demonstration of a Raman fiber distributed feedback laser," in proceedings of CLEO'11, PDPA11 (2011).

[10] C. Fukai, K. Nakajima, J. Zhou, K. Tajima, K. Kurokawa, and I. Sankawa, "Effective Raman gain characteristics in germanium- and fluorine-doped optical fibers," Opt. Lett. 29, 545-547 (2004).

[11] M. Ibsen, M.K. Durkin, M.J. Cole and R.I. Laming, "Sinc-sampled fibre Bragg gratings for identical multiple wavelength operation", Photon. Technol. Lett., 10, 842-844 (1998).

[12] R. Stegeman, L. Jankovic, H. Kim, C. Rivero, G. Stegeman, K. Richardson, P. Delfyett, Y. Guo, A. Schulte, and T. Cardinal, "Tellurite glasses with peak absolute Raman gain coefficients up to 30 times that of fused silica," Opt. Lett. 28, 1126-1128 (2003).

[13] R. Engelbrecht, A. Siekiera, R. Bauer, R. Neumann, and B. Schmauss, "Characterization of Short PM Raman Fiber Lasers with a Small Spectral Bandwidth," in proceedings of OFC/NFOEC'11, paper OMQ2 (2011). 